\title{
Efeitos da Hidroterapia na Capacidade Vital Forçada de Paciente com Esclerose Lateral Amiotrófica
}

\author{
Effects of Hydrotherapy In Forced Vital Capacity of Patients With Amyotrophic Lateral Sclerosis
}

Priscila Santos Albuquerque ${ }^{1}$, Fátima Aparecida Caromano ${ }^{2}$

\section{RESUMO}

Objetivo. Avaliar os efeitos de um programa de hidroterapia na capacidade vital forçada de paciente com Esclerose Lateral Amiotrófica (ELA). Método. Estudo de caso de paciente com diagnóstico de ELA, sexo masculino e 58 anos de idade, que se submeteu a sessões de hidroterapia com ênfase no treinamento dos músculos respiratórios e na mobilidade da caixa torácica, durante 6 meses, 2 vezes por semana, em sessóes com duração de 40 minutos. A avaliação da capacidade vital forçada (CVF \%) foi realizada com espirômetro, antes, durante e após o programa de hidroterapia. Resultados. A CVF, antes do início do programa foi de $100,7 \%$ do predito. Após a realização de 25 sessóes de hidroterapia a CVF demonstrada foi de $69 \%$ do predito. Ao final, na 49a sessão a CVF esteve em 55,5\%. Conclusáo. Apesar da queda da CVF, esperada devido à progressáo da doença, o paciente foi capaz de realizar as sessóes de hidroterapia sem sinais e/ou sintomas de desconforto respiratório. Sugere-se reprodução do estudo em um número maior de sujeitos, utilizando grupo-controle, com o objetivo de gerar indicadores sobre a interferência na capacidade vital forçada de pacientes portadores de ELA.

Unitermos. Hidroterapia, Capacidade Vital Forçada, Doenças Neuromusculares.

Citaçáo. Albuquerque PS, Caromano FA. Efeitos da Hidroterapia na Capacidade Vital Forçada de Paciente com Esclerose Lateral Amiotrófica.

\begin{abstract}
Objective. To evaluate the effects of a hydrotherapy program on forced vital capacity of patients with Amyotrophic Lateral Sclerosis (ALS). Method. Case study of a patient diagnosed with ALS, male and 58 years old, who underwent hydrotherapy sessions with emphasis on training of respiratory muscles and chest mobility, 6 months, 2 times a week, in sessions lasting 40 minutes. Evaluation of forced vital capacity (FVC\%) was performed with spirometer before, during and after the hydrotherapy program. Results. FVC, before the program was $100.7 \%$ of predicted. After conducting 25 sessions of hydrotherapy demonstrated FVC was 69\% predicted. Finally, at the 49th session in FVC was 55.5\%. Conclusion. Despite the decline in FVC, expected due to disease progression, the patient was able to perform hydrotherapy sessions without signs and/or symptoms of respiratory distress. It is suggested reproduction study in a larger number of subjects, using the control group, with the aim of generating indicators on the interference in the forced vital capacity in patients with ALS.
\end{abstract}

Keywords. Hydrotherapy, Forced Vital Capacity, Neuromuscular Diseases.

Citation. Albuquerque PS, Caromano FA. Effects of Hydrotherapy In Forced Vital Capacity of Patients With Amyotrophic Lateral Sclerosis.
Pesquisa realizada na Universidade Sáo Judas Tadeu (USJT) em parceria com a Universidade de Sáo Paulo (USP), Sáo Paulo-SP, Brasil.

1.Fisioterapeuta, Especialista em Hidroterapia nas Doenças Neuromusculares e em Pneumologia pela Universidade Federal de São Paulo (UNIFESP), Mestranda em Ciências da Reabilitação pelo Departamento de Fisioterapia, Fonoaudiologia e Terapia Ocupacional da Faculdade de Medicina da Universidade de São Paulo (FMUSP), São Paulo-SP, Brasil.

2.Doutora, Professora do Curso de Fisioterapia da Faculdade de Medicina da USP, Chefe do Laboratório de Fisioterapia e Comportamento da Faculdade de Medicina da Universidade de São Paulo (FMUSP), Orientadora pelo Programa de Ciências da Reabilitação pelo Departamento de Fonoaudiologia, Terapia Ocupacional e Fisioterapia (FOFITO) da Faculdade de Medicina da Universidade de São Paulo (FMUSP), São Paulo-SP, Brasil.
Endereço para correspondência: FMUSP - Departamento de Fisioterapia, Fonoaudiologia e Terapia Ocupacional A/C Profa. Fátima Aparecida Caromano Rua Cipotânea, 51, Butantã, Cidade Universitária CEP 05360-160, São Paulo-SP, Brasil. E-mail: caromano@usp.br

Relato de Caso Recebido em: 08/10/12 Aceito em: 10/06/13 Conflito de interesses: não 


\section{INTRODUÇÃO}

A ELA acomete o fascículo lateral do corno anterior da medula espinhal decorrente da degeneraçáo dos motoneurônios superiores e/ou motoneurônios inferiores. Um dos principais sintomas da ELA é a fraqueza muscular progressiva devido à atrofia da musculatura. $\mathrm{O}$ comprometimento da musculatura respiratória, ao longo do tempo, faz com que o paciente necessite de ventilação mecânica e higiene brônquica constante ${ }^{1-3}$.

Com o objetivo de minimizar complicaçóes respiratórias que levam a infecçôes frequentes, a fisioterapia é um dos recursos mais utilizados no tratamento e juntamente com a equipe multidisciplinar, visa à melhora da qualidade de vida ${ }^{4-6}$.

Especificamente a fisioterapia aquática, em indivíduos normais, interfere no sistema cardiorrespiratório, devido aos princípios físicos da água. Além disso, devido ao empuxo, o paciente é capaz de desempenhar tarefas que não são possíveis de serem realizadas em solo devido à fraqueza da musculatura ${ }^{7-10}$.

Apesar de a fisioterapia aquática ser um recurso que rotineiramente faz parte do tratamento, poucos estudos descrevem sua influência na capacidade vital forçada. Desta forma, este estudo elaborou um programa de hidroterapia específico para os músculos envolvidos com o sistema respiratório e avaliou seu efeito na função respiratória de um paciente com ELA. Posteriormente, será aplicado em outros pacientes para confirmação dos resultados.

O objetivo deste estudo foi avaliar um programa de hidroterapia para o treinamento dos músculos envolvidos com o sistema respiratório e mobilidade da caixa torácica e avaliar seus efeitos na CVF de paciente com ELA.

\section{MÉTODO}

\section{Seleção da Amostra e Casuística}

No ambulatório de doenças neuromusculares da Universidade São Judas Tadeu (USJT), um grupo de fisioterapeutas, previamente orientados quanto à fisiopatologia da doença, selecionou o prontuário de 6 pacientes com diagnóstico confirmado de ELA na forma clássica.

Desta amostra, somente 1 assinou um termo concordando em participar de pesquisas clínicas, enquanto estivesse em tratamento na clínica escola da Universidade São Judas Tadeu (USJT).
Participou deste estudo 1 paciente com diagnóstico confirmado de ELA na forma clássica, do sexo masculino, 58 anos, altura de $1,67 \mathrm{~m}$ e peso de $76 \mathrm{Kg}$. O mesmo assinou o TCLE, concordando em participar da pesquisa.

\section{Intervenção}

O tratamento foi realizado na Clínica Escola da USJT, em piscina aquecida de $33^{\circ}$ a $35^{\circ} \mathrm{C}$ e profundidade de $1,28 \mathrm{~m}$.

O programa teve duração de 6 meses com frequência de 2 vezes por semana, duração de 40 minutos cada sessão, totalizando 49 atendimentos.

Para evitar tendência nos resultados, o fisioterapeuta que aplicou o programa de hidroterapia não participou dos testes de espirometria.

O paciente foi orientado quanto aos sinais e sintomas de fadiga e para avisar o fisioterapeuta, realizar período de repouso imediato e só retomar a atividade após melhora dos sintomas.

Visto que o paciente já possuía experiência prévia em piscina, não foi necessário realizar um programa de adaptação ao meio aquático.

As sessôes foram baseadas nas técnicas de facilitação neuromuscular proprioceptiva (PNF), Reeducação Postural Global (RPG), Método Halliwick e cinesioterapia para membros superiores. Não foi estabelecido o número de repetiçóes e tempo para cada atividade, pois ao primeiro sinal de fadiga foi realizado período de repouso.

Durante o atendimento o paciente manteve o tórax em imersão com a água no nível do pescoço, aumentando em $60 \%$ o trabalho dos músculos respiratórios devido à pressão hidrostática.

O Programa de Hidroterapia:

- Na postura sentada: exercícios de conscientização corporal e alinhamento postural com e sem efeito metacêntrico e turbulência (anterior e posterior) (10 minutos), facilitação neuromuscular proprioceptiva para membros superiores nas diagonais funcionais (10 minutos), exercícios ativos livres de membros superiores com velocidade máxima suportada pelo paciente (10 minutos), controle respiratório oral e nasal (5 minutos).

- Em flutuação: relaxamento com mobilização do tórax (5 minutos). 
Avaliação Respiratória:

Para mensurar a função respiratória do paciente, a CVF foi avaliada em solo, antes, durante ( $25^{\text {a }}$ sessão) e após a realizaçáo do programa de hidroterapia, sem a realização de quaisquer tipos de atividade física durante este período.

A CVF foi avaliada com o espirômetro do tipo Spirocard e de acordo com o Consenso de Espirometria foi escolhida a melhor de três medidas na postura sentada, com predito estabelecido por NHANES.

Foi solicitado ao paciente uma inspiração máxima e a exalação do ar com o máximo esforço. Todas as medidas foram realizadas pelo mesmo fisioterapeuta garantindo padronização do comando verbal e estímulo para realização do teste.

\section{RESULTADOS}

A CVF antes do início do programa (tempo 0) foi de $100,7 \%$ do predito e após a realização de 25 sessóes de hidroterapia (tempo 1) a CVF passou a 69\% (Figura 1). Ao final, na $49^{a}$ sessão (tempo 2) a CVF foi para 55,5\%.

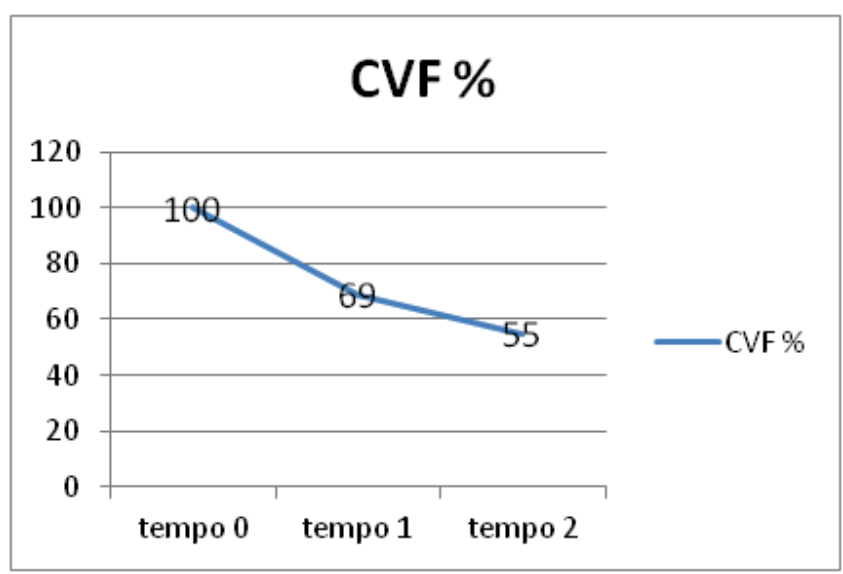

Figura 1. A Capacidade Vital forçada (CVF) antes do início do programa (tempo 0), após a realização de 25 sessôes de hidroterapia (tempo 1) e na $49^{\mathrm{a}}$ sessão (tempo 2).

\section{DISCUSSÃo}

A forma mais prevalente de ELA é conhecida como clássica e se caracteriza por apresentar sinais referentes à lesão de neurônio motor inferior (amiotrofia), neurônio motor superior (espasticidade) e bulbo (disartria/disfagia). Neste estudo, apesar do diagnóstico de ELA na forma clássica, o paciente não apresentou sinais de espastici- dade relatando sintomas relacionados ao bulbo, ou seja, disfagia e insuficiência respiratória.

A insuficiência respiratória na ELA se deve principalmente a perda de força da musculatura envolvida com o sistema respiratório e se agrava pelo acúmulo de secreçôes e broncoaspiração. Normalmente a ventilação não invasiva é introduzida antes que haja insuficiência respiratória, visando prevenir infecçóes recorrentes. O paciente deste estudo não fazia uso de ventilação mecânica, pois as espirometrias trimestrais de controle da área médica e os sinais de desconforto respiratório ainda não indicavam seu uso.

Devido à ausência de informaçôes sobre programas de hidroterapia em pacientes portadores de ELA, este estudo é importante e poderá ser reproduzido em grupos para geração de resultados. Além disso, foi elaborado um modelo de programa de hidroterapia com ênfase nos músculos envolvidos com o sistema respiratório e mobilidade da caixa torácica, podendo ser útil para outros pacientes com patologias que comprometem este sistema.

Um estudo realizado em 2009 demonstrou a importância da reabilitação aquática como recurso terapêutico para tratamento dos pacientes com ELA. Foram avaliados 2 pacientes com diagnóstico de ELA definida. O programa de hidrocinesioterapia durou 16 semanas, com sessóes de 45 minutos, duas vezes por semana em piscina aquecida a $33^{\circ} \mathrm{C}$. Foram avaliadas, no início e término do estudo: qualidade de vida, funcionalidade, força muscular, fadiga e dor ${ }^{11}$.

Considerando-se que a ELA é uma doença degenerativa incurável com características de rápida progressão, foi concluído que a hidrocinesioterapia obteve bons resultados na qualidade de vida, funcionalidade, força muscular, fadiga e dor. Esta pesquisa, diferentemente de nosso estudo, não abordou a avaliação da capacidade respiratória no portador de ELA.

Não foram utilizadas escalas de avaliação, pois não existem instrumentos validados para avaliação de portadores de ELA em piscina. O uso destes instrumentos poderia gerar dados subjetivos.

Maiores discussôes ficam prejudicadas, uma vez que, existem poucos artigos na literatura que discutem os efeitos e benefícios de um programa de hidroterapia em pacientes com ELA. 


\section{CONCLUSÃO}

Apesar da queda da CVF, esperada devido à doença, o paciente foi capaz de realizar as sessóes de hidroterapia sem sinais e/ou sintomas de desconforto respiratório. Sugere-se reprodução do estudo em um número maior de sujeitos, utilizando grupo-controle, com o objetivo de gerar indicadores sobre a interferência na capacidade vital forçada de pacientes portadores de ELA.

\section{REFERÊNCIAS}

1.Van den Berg JP, Kalmijn S, Lindeman E, Veldink JH, de Visser M, Van der Graaff MM, et al. Multidisciplinary ALS care improves quality of life in patients with ALS. Neurology 2005;65:1264-7.

http://dx.doi.org/10.1212/01.wnl.0000180717.29273.12

2.Miller RG, Rosenberg JA, Gelinas DF, Mitsumoto H, Newman D, Sufit $\mathrm{R}$, et al. Practice parameter: the care of the patient with amyotrophic lateral sclerosis (an evidence-based review): report of the Quality Standards Subcommittee of the American Academy of Neurology: ALS Practice Parameters Task Force. Neurology 1999;52(7):1311-23.
http://dx.doi.org/10.1212/WNL.52.7.1311

3.Bertorini TE (ed.). Clinical evaluation and diagnostic tests for neuromuscular disorders. Woburn: Butterworth-Heinemann; 2002, p.50-53.

4.Bradley WG, Anderson F, Bromberg M, Gutmann L, Harati Y, Ross M, et al. Current management of ALS: comparison of the ALS CARE Database and the AAN Practice Parameter. The American Academy of Neurology. Neurology 2001;57(3):500-4.

http://dx.doi.org/10.1212/WNL.57.3.500

5.Cronin S, Hardiman O, Traynor BJ. Ethnic variation in the incidence of ALS: a systematic review. Neurology 2007;68(13):1002-7.

http://dx.doi.org/10.1212/01.wnl.0000258551.96893.6f

6.Dubrovsky AL, Sica RE. Current treatment pathways in ALS: a South American perspective. Neurology 1999;53(8 Suppl 5):S11-6.

7.Ruoti RG, Morris DM, Cole AJ. Reabilitação Aquática. São Paulo: Manole, 2000, 463p.

8.Jakaitis F. Reabilitação e terapia aquática: aspectos clínicos e práticos. São Paulo: Roca, 2007, 282p.

9.Campion MR. Hidroterapia: princípios e prática. São Paulo: Manole, 2000, $332 \mathrm{p}$.

10.Schanzer GS, Queiroz SS. Fisioterapia aquática aplicada à neurologia. In: Sacchelli T, Accacio LMP, Radl ALM. Fisioterapia aquática. Barueri: Manole; 2007.p.191-202.

11.Cunha MCB, Labronici RHDD, Oliveira ASB, Gabbai AA. Relaxamento aquático, em piscina aquecida, realizado pelo método AI CHI: Uma nova abordagem hidroterapêutica para pacientes portadores de doenças neuromusculares. Rev Neurocienc 2000;8:47-49. 